\title{
AN ACCRETION DISK MODEL FOR THE INNERMOST 200PC OF THE GALAXY
}

\author{
W.J. DUSCHL \\ Institut für Theoretische Astrophysik, Universität Heidelberg, \\ Im Neuenheimer Feld 561, W-6900 Heidelberg, Germany \\ S.v. LINDEN and P.L. BIERMANN \\ Max-Planck-Institut für Radioastronomie, Auf dem Hügel 69, \\ W-5300 Bonn, Germany
}

\begin{abstract}
We demonstrate that it is possible to understand observed radial velocities of molecular clouds close to the Galactic Center as being due to motion of this material in an accretion disk. Our models indicate a stationary disk with a radial mass flow rate of $10^{-1.8} \mathrm{M}_{\odot} / \mathrm{yr}$ with a viscosity of $1.9 \cdot 10^{3} \mathrm{pc} \mathrm{km} / \mathrm{s}$ at $100 \mathrm{pc}$ from the Galactic Center and a radial dependence of it, that goes approximately like the square root of the radius from the Galactic Center.
\end{abstract}

\section{MODEL}

Observations give two geometrical coordinates (galactic longitude and latitude) and one component of the velocity vector (radial velocity). One needs a physical model for the dynamics or the observed matter in order to gain information about the other two velocity components and, especially, the third geometrical coordinate. In our model, we assume that the overall flow patterns close to the Galactic Center (GC) are well described in the framework of an accretion disk picture. We interpret the molecular clouds as being prominent tracers of the local velocities. As we restrict our analysis to accrection disks that are geometrically thin in the direction perpendicular to its rotation plane, we do not resolve this direction. In this way, only four of the orginally six dimensions of phase space are dealt with in our analysis. We allow for a non stationary disk with radially variable viscosity. The disk is assumed to evolve in the gravitational potential of a sperically symmetric but not necessarily point-like mass distribution. For this mass distribution, we use the values as given by Genzel and Townes (1987, Ann.Rev.Astron.Astrophys. 25, 377). In the case of non-negligible self-gravity in the disk, we take star formation into account; stars formed out of the disk are assumed to decouple from the disk and thus do no longer contribute to the accretion. Further details of the models are described elsewhere (Linden, Dusch1, and Biermann, 1992, Astron.Astrophys., in press). 


\section{OBSERVATIONS}

For our models, we use the ${ }^{13} \mathrm{CO}$ observations of the molecular cloud M-0.130.08 (Zylka et al., 1990, Astron.Astrophys. 234, 133) and $\mathrm{H}_{2} \mathrm{CO}$ observations ot the $-190 \mathrm{~km} / \mathrm{s}$ features on either side of the GC (Pauls et al., 1992, Astrophys.J., in press). The set encompasses altogether nine observations. Our aim is to determine the missing geometrical coordinate from this position-velocity observations with the help of the accretion disk model. Moreover, this will give us information about the physical conditions in the GC accretion flow.

\section{RESULTS}

We find that the observations are best explained by modelling it with a stationary accretion disk with a radial mass flow rate of $10^{-1.8} \mathrm{M}_{\odot} / \mathrm{yr}$. Additional vertical mass infall into the disk does not improve our solutions, but we cannot exclude it as long as its total rate (integrated over the entire disk) is small compared to the aforementioned rate. For the viscosity we find a value of $1.9 \cdot 10^{3}$ $\mathrm{pc} \mathrm{km} / \mathrm{s}$ at a radius of $100 \mathrm{pc}$ from the GC. Its radial variation is proportional to $\mathrm{s}^{0.45}$ ( $\mathrm{s}$ : radial distance from the $\mathrm{GC}$ ). While the value of the viscosity is very high compared to what a standard accretion disk (Shakura and Sunyaev, 1973, Astron.Astrophys., 24, 337) would give, it is in good agreement with observed velocity dispersion and scale height. This topic is addressed in detail by Linden, Biermann, Lesch, Schmutzler, and Duschl (1992, submitted to Astron.Astrophys.) and by Biermann, Duschl, and Linden (1992, submitted to Astron.Astrophys.). Disks with these parameters are not self-gravitating; thus star formation does not play an importent rôle. We find that viscosity and mass flow rate are the two most important parameters for our models. This allows to determine both parameters very accurately (much better than half an order of magnitude). In our models, M-0.13-0.08 is found to be located $115 \mathrm{pc}$ from the $\mathrm{GC}$ on the near side. The $-190 \mathrm{~km} / \mathrm{s}$ features lie at a $\mathrm{GC}$ radius of $10 \ldots 15 \mathrm{pc}$ on the far side.

\section{CONCLUSIONS}

It is justified to use molecular clouds as tracers of the flow pattern close to the GC. An accretion disk model gives a good representation of the observations. We regard this result as a first step towards mapping the GC region.

\section{ACKNOWLEDGMENTS}

Part of this work was supported by the Deutsche Forschungsgemeinschaft (WJD, SvL: SFB328) and through a Verbungforschungs-grant (WJD: PH/055HD45A). 